\title{
Regionalentwicklung unter Schrumpfungsbedingungen
}

Herausforderung für die Raumplanung in Deutschland

\section{Regional Development in Conditions of Shrinkage}

\author{
The challenge for spatial planning in Germany
}

\section{Kurzfassung}

Demographische Schrumpfung ist ein Fakt in Deutschland und in Europa. Sie führt in Ostwie in Westdeutschland zu gravierenden Veränderungen räumlicher Strukturen und zu neuen Herausforderungen für die Raumentwicklung. Dabei ist zu berücksichtigen, dass sich Schrumpfung und Wachstum häufig auf engstem Raum nebeneinander finden lassen. Vor diesem Hintergrund beschäftigt sich der Beitrag mit der Frage nach notwendigen Veränderungen der Raumplanung und nach ihren Steuerungsperspektiven. Das Zusammenspiel einer Rahmensteuerung "von oben" und einer Koordination „von unten" im Sinne einer anreizorientierten Mehrebenensteuerung - so ein Fazit - könnte dabei helfen, differenzierter als bisher auf die Kleinteiligkeit von Entwicklungsprozessen zu reagieren.

\begin{abstract}
Demographic shrinkage is a fact of life both in Germany and in Europe. In both eastern and western Germany it is leading to dramatic changes in spatial structures and throwing up new challenges for spatial planning. It is, however, important to note that shrinkage and growth can often be found side by side and in extremely close proximity to each other. It is against this background that the author explores the changes which need to be made to spatial planning and possible perspectives for steering development. The combination of "top-down" framework steering and "bottom-up" co-ordination - i.e. an incentive-oriented, multi-tiered form of steering - could, it is argued, be a helpful approach to facilitate more differentiated reactions than have previously been possible to the local particularities of development processes.
\end{abstract}

\section{Vorsicht Schrumpfung?}

Kaum ein Begriff wurde in letzter Zeit so kontrovers diskutiert wie der Begriff der Schrumpfung. Schrumpfung signalisiere negative Assoziationen und sei insbesondere auch angesichts der ohnehin bestehenden "Transformationslücke" in Deutschland politisch unbrauchbar, argumentieren die einen. Man müsse Realitäten und die damit verbundenen Probleme offen benennen, sagen hingegen die anderen.

Gleichwohl ist Schrumpfung heute ein Fakt in Deutschland. Allerdings wird häufig darauf verzichtet, näher zu beschreiben, was im Einzelfall konkret unter Schrumpfung verstanden wird. Zudem werden nur selten die räumlichen Auswirkungen von Schrumpfung thematisiert. Auch die Tatsache, dass es bei Schrumpfungsprozessen Gewinner und Verlierer gibt, dass es zu räumlichen Polarisierungen kommt und dass die Thematik eine enorme gesellschaftliche Sprengkraft besitzt, wird kaum behandelt.

Dabei ist die Thematik nicht neu. Bereits in den 70er und 80er Jahren wurde von wissenschaftlicher Seite auf dramatische demographische Schrumpfungsprozesse hingewiesen (u.a. Mackensen 1984), allerdings kam es seinerzeit nicht zu einer Auseinandersetzung mit dem Schrumpfungsthema. Und der deutsche Vereinigungsprozess setzte in der Folgezeit andere Schwerpunkte in der öffentlichen Debatte. 
Demographische Schrumpfung ist heute nicht nur ein deutsches, sondern ein europäisches Phänomen (vgl. Abb. 1). Nach Angaben des Zweiten Kohäsionsberichts der Europäischen Kommission (Europäische Kommission 2001, S. 41 ff.) nimmt die Bevölkerung im Zeit- raum von 2000 bis 2010 in vielen Regionen Deutschlands, Italiens, Frankreichs, Großbritanniens und Österreichs ab. Bis zum Jahr 2025 wird sogar ein Bevölkerungsrückgang in 90 der 200 NUTS-2-Regionen erwartet. Betroffen sind nahezu alle Mitgliedstaaten.

\section{Abbildung 1}

Bevölkerungsentwicklung in europäischen Großstädten (1981-1996)

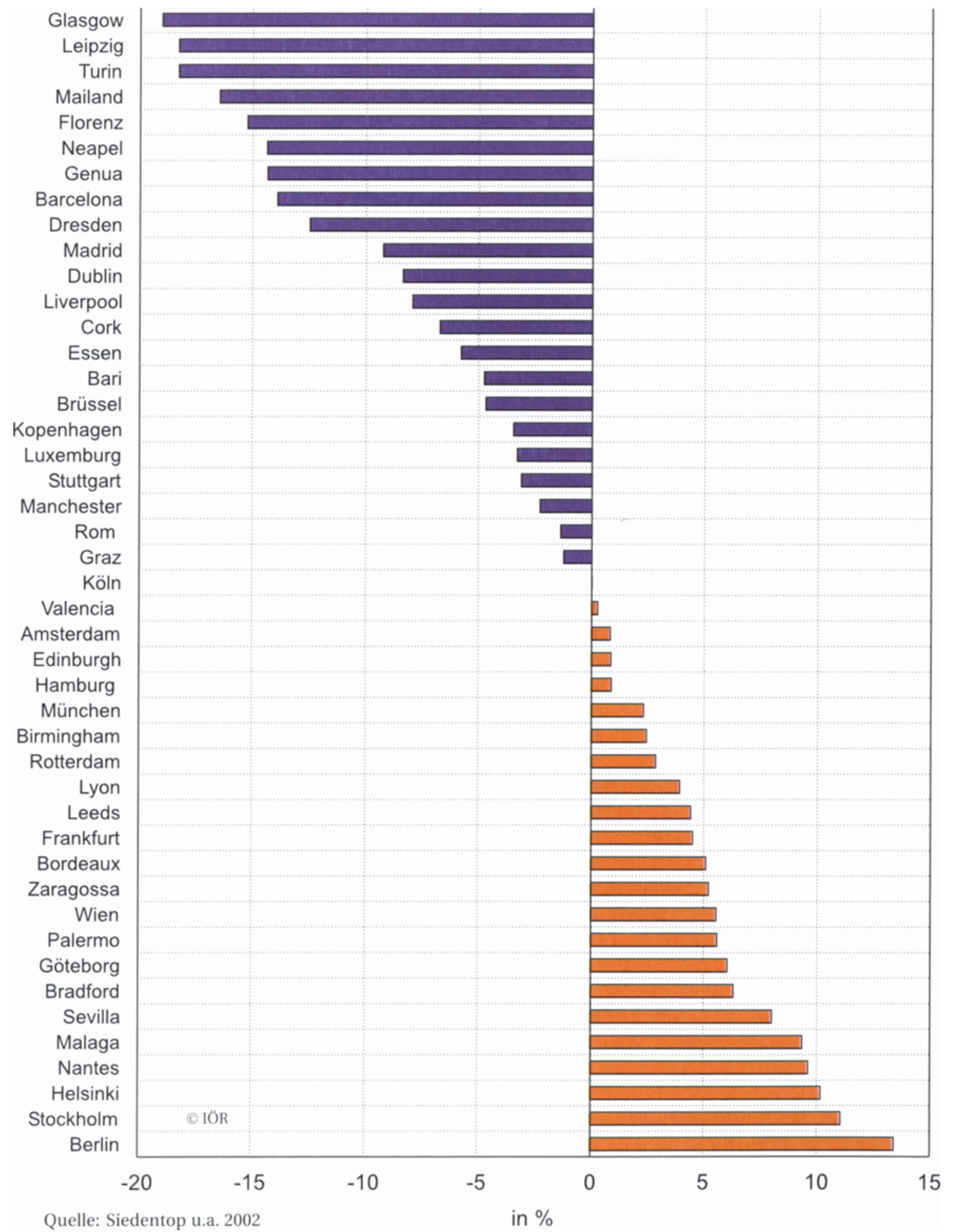




\section{Zum Schrumpfungsbegriff}

Grundsätzlich kann der Begriff der Schrumpfung als negative Veränderung einer Variablen begriffen und dem Begriff des Wachstums als positiver Veränderung dieser Variablen gegenübergestellt werden. Daher muss jeweils geklärt werden, auf welche Variablen sich der Schrumpfungsbegriff bezieht.

In den Raumwissenschaften wird der Begriff häufig im Sinne eines anhaltenden Rückgangs der Bevölkerung verwendet (vgl. Winkel 2001). Er bezeichnet ein bestimmtes Prozessmuster, das Auswirkungen in den verschiedenen Sektoren der Stadt- und Regionalentwicklung entfaltet und Reaktionen der betroffenen Akteure hervorruft. Schrumpfung kann dabei sehr wohl mit wirtschaftlichem Wachstum verknüpft sein und darf nicht unreflektiert als allgemeiner wirtschaftlicher, demographischer und sozialer Niedergang einer Region aufgefasst werden.

Wood (1994) hingegen versteht unter Schrumpfung einen mehrdimensionalen Prozess und eine krisenhafte Umstrukturierung von Bevölkerung, Wirtschaft und in deren Folge auch der baulich-physischen Strukturen. Während kurzfristige Schwankungen - zum Beispiel auf Grund von konjunkturbedingter Migration in einer Gesellschaft zum Normalfall gehören, deutet Schrumpfung in diesem Sinne auf einen außerordentlichen und komplexen Handlungsbedarf bei der Veränderung gesellschaftlicher Strukturen hin.

Wenngleich vieles für die Sichtweise von Wood spricht, so bildet in diesem Beitrag doch die demographische Entwicklung den Ausgangspunkt der Überlegungen. Sie wird im Folgenden näher erläutert und in ihren Beziehungsgeflechten und Konsequenzen diskutiert. Insbesondere wird auf die Herausforderungen der Raumwissenschaften einzugehen sein, ihre primär für Wachstumsprozesse entwickelten Aussagen zum planerischen Instrumentarium (zusammenfassend ARL 1998) unter Berücksichtigung eines anhaltenden Bevölkerungsrückgangs und mit Blick auf die komplexen und dynamischen Wirkungszusammenhänge innerhalb und zwischen den Sektoren der Stadt- und Regionalentwicklung systematisch zu prüfen und weiterzuentwickeln.

\section{Räumliche und sektorale Differenzierung von Schrumpfung}

Schrumpfung hat räumlich und zeitlich unterschiedliche Ausprägungen. Die Bevölkerungszahlen sanken in den 90er Jahren vor allem in Ostdeutschland auf breiter Front. Einen Bevölkerungsrückgang gab es in Ostdeutschland aber auch schon vor der Wende. Zwischen
1980 und 1997 nahm die Bevölkerung um $6 \%$ ab. Rückläufige natürliche Zuwachsraten sind bereits seit den 70er Jahren festzustellen. Regional überlagert wurden und werden diese allerdings durch Wanderungsbewegungen.

In Ostdeutschland verloren im Zeitraum 1990 bis 1999 sowohl die größeren Städte als auch die kleineren Gemeinden an Bevölkerung. In Westdeutschland hingegen ergab sich ein Bevölkerungsrückgang vor allem für die großen Städte, wie München, Stuttgart oder die Städte des Ruhrgebiets. Schrumpfungserscheinungen in Ostdeutschland zeigen allerdings kein einheitliches Bild, sondern weisen eine Vielzahl regionaler und lokaler Differenzierungen auf. $\mathrm{Zu}$ unterscheiden ist insbesondere zwischen ländlich-peripheren Regionen, z.B. den Grenzräumen Sachsens zu Tschechien und Polen oder im Nordosten von Mecklenburg-Vorpommern einerseits und den Stadtregionen andererseits. Bevölkerungsrückgänge sind in den Kernstädten in erheblichem Maße auf Stadt-Umland-Wanderungen zurückzuführen, die zu neuen Mustern der intraregionalen Bevölkerungsverteilung geführt haben.

Im Vergleich zu Westdeutschland sind die ostdeutschen Stadtregionen durch zwei Besonderheiten gekennzeichnet:

(1)

Die intraregionale Bevölkerungsumverteilung ergibt sich bei insgesamt rückläufiger Bevölkerung, was interkommunale Verteilungskämpfe um Bevölkerungszuwächse verstärken kann. In westdeutschen Stadtregionen, wie München oder Stuttgart, sind hingegen auf regionaler Ebene noch Bevölkerungsgewinne zu verzeichnen. Auch in einigen westdeutschen Stadtregionen wird allerdings bis zum Jahr 2025 mit Bevölkerungsrückgängen zu rechnen sein.

(2)

Infolge des politischen Strukturwandels änderten sich die sozioökonomischen Rahmenbedingungen ostdeutscher Stadtregionen innerhalb kürzester Zeit. Auch fanden bzw. finden die anschließenden Transformationsprozesse (Deindustrialisierung, soziale Segregation, Flächenverbrauch usw.) quasi im Zeitraffertempo statt.

Es ist zu erwarten, dass Schrumpfung den lokalen und regionalen Akteuren, wie z.B. Kommunen und Wohnungsunternehmen, eine Neuorientierung bei der Erfüllung ihrer Aufgaben abverlangt. Anzeichen hierfür zeigen sich in der Kommunalpraxis, wenn ostdeutsche Städte, wie Leipzig oder Dresden, eine Abkehr von herkömmlichen, durch westdeutsche Stadtregionen geprägte Dichtevorstellungen vollziehen und ihre spezifischen Entwicklungspotenziale im Wettbewerb mit den 
Umlandgemeinden zu nutzen suchen (z.B. verstärkte Integration von Stadthäusern und weiteren einfamilienhausähnlichen Wohnformen in den städtischen Wohnungsbestand, Durchgrünung von innerstädtischen Wohngebieten, großräumige Vernetzung von Freiflächen).

\section{Entwicklungstendenzen und ihre Konsequenzen}

\section{Bevölkerungsentwicklung und ihre Auswirkungen}

Demographische Schrumpfung und demographisches Wachstum liegen in Deutschland räumlich meist nah beieinander. Die weitaus überwiegende Zahl der Gemeinden in der Bundesrepublik hatte in den 90er Jahren Wachstum zu verzeichnen. Im Agglomerationsraum Saar wuchsen $69 \%$, im Agglomerationsraum München $96 \%$ aller Gemeinden (vgl. Abb. 2). Im Agglomerationsraum Berlin verzeichneten $53 \%$ der Gemeinden eine Bevölkerungszunahme, was insgesamt $\mathrm{zu}$ einem moderaten Wachstum der Region von knapp $1 \%$ führte. Im Agglomerationsraum des „Sachsendrei- ecks“ (Dresden - Leipzig/Halle - Chemnitz/Zwickau) wuchsen $42 \%$ der Gemeinden. Dabei handelte es sich vor allem um die meist kleineren Gemeinden im Umland der großen Städte. Insgesamt kam es hier aber zu einem Bevölkerungsrückgang von etwa $7,5 \%$.

Ebenso wie das Wachstum ist Schrumpfung kein ausschließlich auf einen Teil Deutschlands beschränkter Trend. Schrumpfende Gemeinden finden sich zwar überwiegend, aber nicht ausschließlich in Ostdeutschland. In Ostdeutschland schrumpfen mit Ausnahme des relativ breiten Siedlungsrings um Berlin sowie des „Speckgürtels“ um die übrigen großstädtischen Zentren fast alle Regionen. In Westdeutschland gibt es schrumpfende und alternde Regionen vor allem im Ruhrgebiet, im Saarland und in einigen peripheren Räumen. Bei der Bevölkerungsentwicklung in Ostdeutschland ist $\mathrm{zu}$ beachten, dass Schrumpfung hier zunächst ein Problem der natürlichen Bevölkerungsentwicklung ist. Dieses wird erst in zweiter Linie von Problemen der Abwanderung überlagert. Teilweise, so zum Beispiel im Agglomerationsraum Berlin, kompensiert Zuwanderung die negativen natürlichen Bevölkerungstrends.

\section{Abbildung 2}

Wachsende und schrumpfende Gemeinden in Deutschland (1990 - 1999)
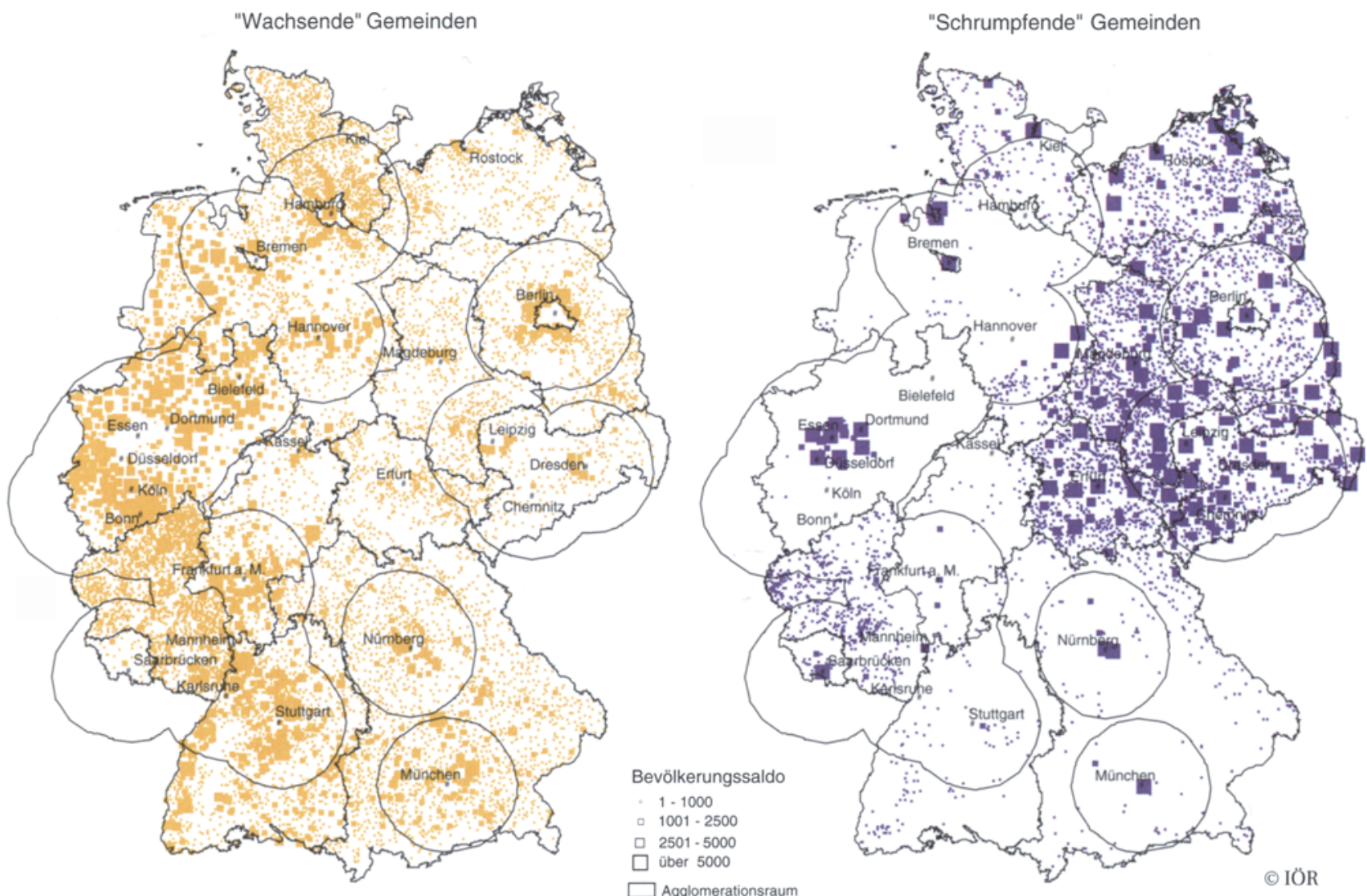

Quelle: Siedentop u.a. 2002 
Abbildung 3

Gemeinden in Deutschland ohne zentralölrtlichen Status mit einem Bevölkerungswachstum von mehr als $20 \%$ (1990-1999)

Agglomerationsraum

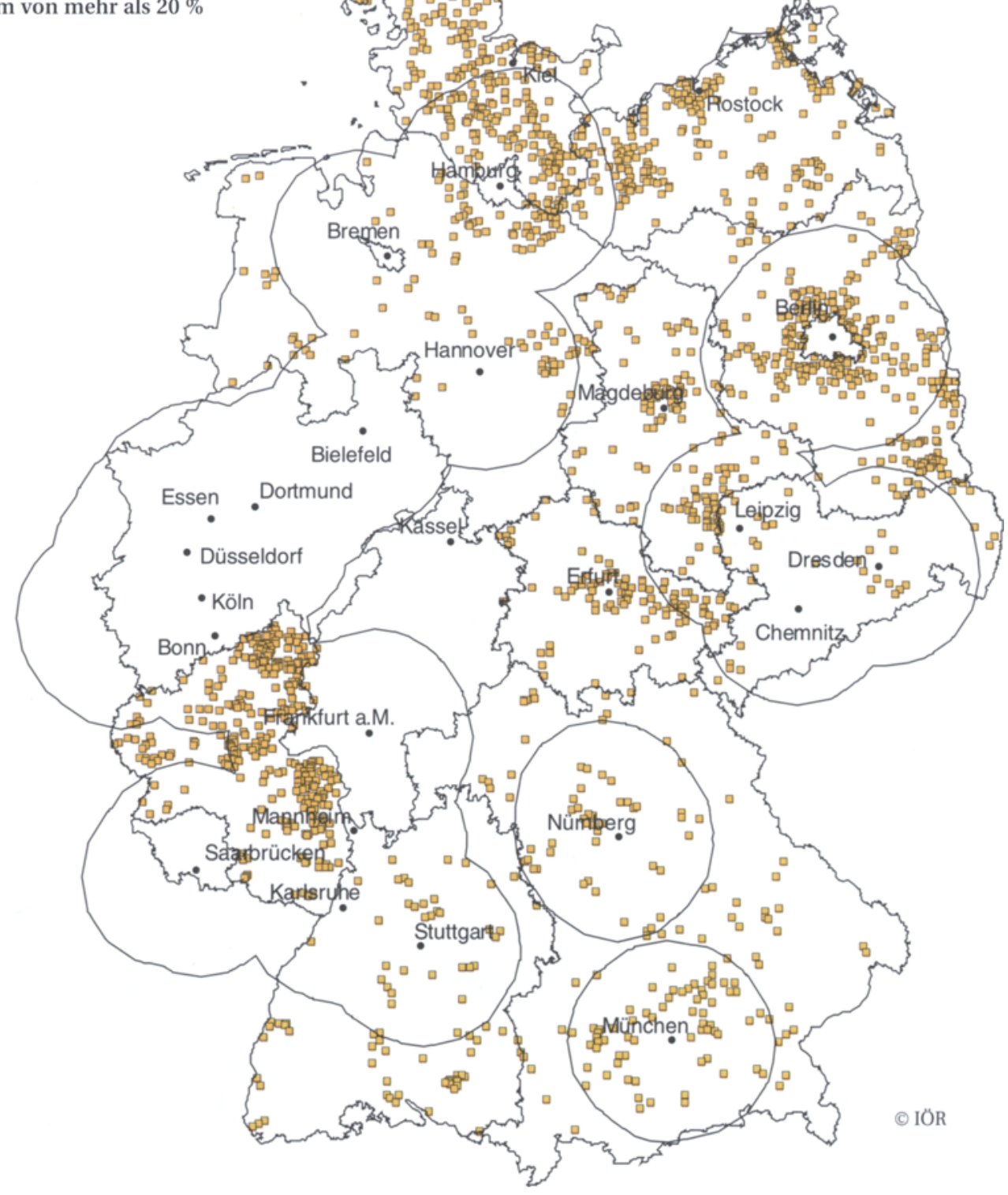

Die Rolle der Raumordnung zur Steuerung von Wachstumstrends kann in den 90er Jahren nur bedingt als erfolgreich angesehen werden. Dies zeigt die bundesweit hohe Zahl an Gemeinden ohne zentralörtliche Funktion mit einem Bevölkerungswachstum von mehr als $20 \%$, das auch bei einer liberalen Position deutlich über einer Eigenentwicklung gelegen haben dürfte (vgl. Abb. 3). Insbesondere die Länder, in denen man die Siedlungsentwicklung über das zentralörtliche System aktiv zu steuern versuchte, weisen eine große Zahl von Gemeinden ohne zentralörtlichen Status mit einem hohen Wachstum auf.
Die kommenden Jahre werden nicht von einer Entwarnung geprägt sein. Die Raumordnungsprognose 2015 des Bundesamtes für Bauwesen und Raumordnung (BBR) hat gezeigt, dass Kernstädte in Ost und West schrumpfen und ländliche Kreise in Agglomerationsräumen in Ost und West wachsen werden. Erhebliche Probleme werden die Mittelstädte in Ostdeutschland (Kernstädte in verdichteten Räumen) haben (vgl. Abb. 4). Zudem wird die Situation in der Zukunft vor allem dort problematisch, wo eine geringe Siedlungsdichte sowie ein starker Bevölkerungsrückgang zu verzeichnen sind, da hier die Infrastrukturkosten überpro- 


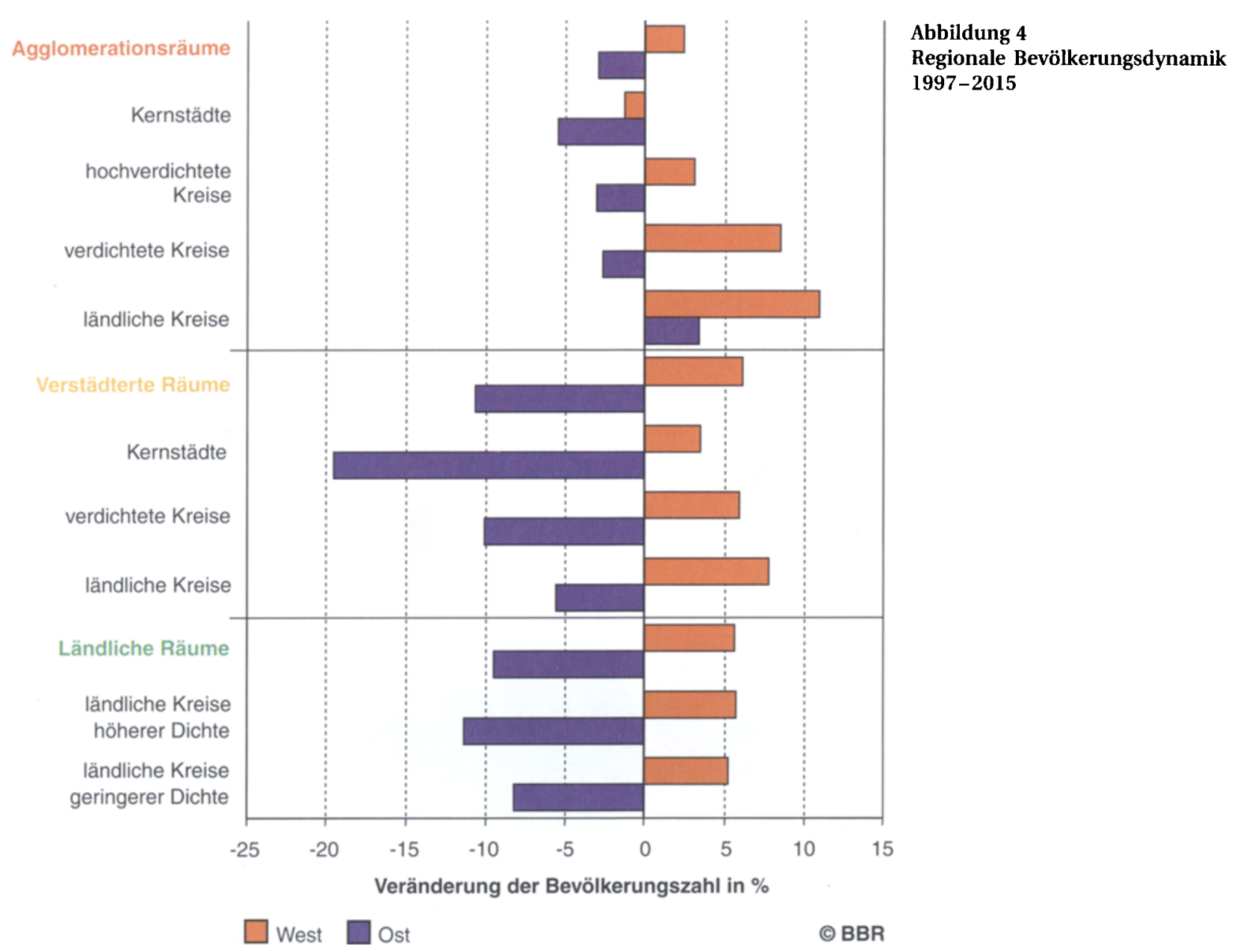

portional hoch sein werden (vgl. Abb. 5). Insgesamt nimmt die Polarisierung zu. Zum West-Ost-Gefälle treten allerdings kleinräumige Disparitäten in Ost und West.

Gravierende Veränderungen - insbesondere in Ostdeutschland - sind durch den Alterungsprozess der Bevölkerung zu erwarten. Zwischen 1997 und 2015 wird der Prognose des BBR zufolge mit einem Anstieg der über 75-Jährigen um fast $62 \%$ und mit einem Rückgang der Kinder und Jugendlichen um 43-45\% gerechnet. Die Zahl der 20- bis 40 -Jährigen wird um über $20 \%$ abnehmen. Die Bevölkerungspyramide wird damit zum Pilz bzw. zum Baum.

Mit diesen Entwicklungen sind erhebliche Konsequenzen verbunden. Vor allem fünf Aspekte sollen hier stichpunktartig genannt werden:

(1)

Durch die steigende Anzahl an Kleinhaushalten, Senioren, Unmobilen kommt es zu qualitativen und strukturellen Bedarfsveränderungen hinsichtlich Infrastruktur und Dienstleistungen.
(2)

Flächenentwicklung und Siedlungsstruktur werden sich gravierend ändern. In dispersen Siedlungsstrukturen wird die Dichte weiter abnehmen, in schwach strukturierten, peripheren Räumen kann es zu einer Welle von "Wüstungen“ kommen, die "öchrige“ Stadt wird zu einem Gegenentwurf der "Zwischenstadt", Innenentwicklung wird ein zunehmend brisantes Thema werden.

(3)

Infrastrukturkosten steigen insbesondere in den dünn besiedelten Regionen und in den interaxialen Zwischenräumen überproportional an, in einigen Infrastrukturbereichen werden vermehrt Unterauslastungen auftreten. Dadurch sinkt deren Rentabilität. Es kann dabei auch zu Funktionsstörungen bei $\mathrm{zu}$ geringer Nutzungsdichte (z.B. im Abwasserbereich) kommen. Einrichtungen stoßen an die Grenzen der Finanzierbarkeit (Schulen, Kultureinrichtungen, Sozialeinrichtungen). In anderen Bereichen (insbesondere für Senioren) wird der Bedarf steigen, ihre Finanzierbarkeit wird jedoch immer schwieriger. 


\section{Abbildung 5}

Überlagerung von Gebieten mit geringer Siedlungsdichte (1996) und Schrumpfungstendenzen (1996-1999)

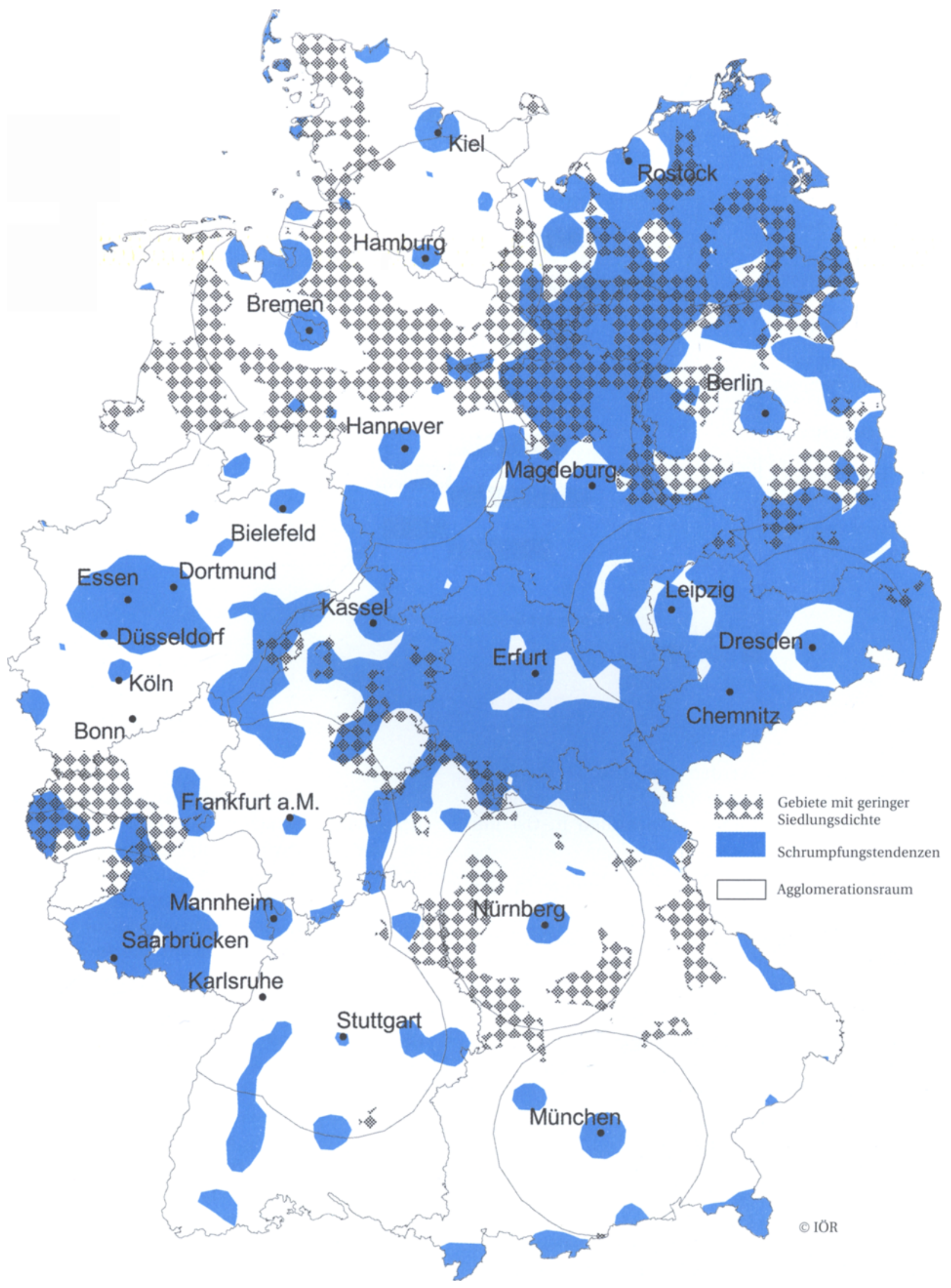


(4)

Im Bereich der kommunalen Finanzen werden sinkende Einnahmen weitgehend gleichbleibenden Ausgaben gegenüberstehen. Gebühreneinnahmen werden sinken (Infrastruktur). Sozialaufwendungen werden steigen. Gleichzeitig müssen Kommunen auf die sich verändernden Formen der Bedarfsdeckung reagieren, was zusätzliche Kosten verursacht.

(5)

Gesellschaftliche Integrationsaufgaben werden erheblich ansteigen. Dies betrifft nicht nur Aufgaben der Integration der Generationen (z.B. durch soziale und altersmäßige Durchmischung von Wohngebieten), sondern vor allem auch der interkulturellen Integration.

\section{Auswirkungen auf den Wohnungsmarkt}

Auf dem Wohnungsmarkt gab es nicht nur in Westdeutschland, sondern auch in Ostdeutschland in den 90er Jahren keine Schrumpfung. Vielmehr waren erhebliche Zuwächse zu verzeichnen. Damit verbunden war nicht selten ein „Speckgürtelsyndrom“, das Phänomen wachsender Kommunen im Umland der Städte. Allerdings holten die Kernstädte Im Hinblick auf ihre Attraktivität als Wohnstandort in der zweiten Hälfte der 90er Jahre erheblich auf, nachdem der größte Teil der Restitutionsfälle gelöst war.

Die Ursachen für das schnelle Wachstum der Wohnungsmärkte in Ostdeutschland sind bekannt: Einerseits bestand erheblicher Nachholbedarf. Haushalte verkleinerten sich, die Nutzung von Wohnungen durch mehrere Familien, die auf Grund von Wohnungsnot vor der Wende nicht unüblich war, führte zu einem zusätzlichen Nachfragepotenzial nach Wohnungen. Hinzu kam die Zunahme des individuellen Flächenbedarfs auf Grund veränderter Lebensstile und Prioritätensetzungen. Andererseits waren die Sonderabschreibungen für Investitionen in den neuen Bundesländern ein wesentlicher Motor der Ankurbelung des Wohnungsmarkts.

Infolge der zunächst boomenden und nur wenig am langfristigen Bedarf orientierten Wohnungsmarktentwicklung ist seit Ende der 90er Jahre in vielen Gemeinden Ostdeutschlands Wohnungsleerstand an der Tagesordnung. In den ostdeutschen Ländern betrug die Wohnungsleerstandsquote Ende 2000 mit ungefähr 1 Mio. Wohneinheiten etwa $13 \%$. Groß- und Mittelstädte in Sachsen verzeichneten zwischen 15 und $25 \%$ Leerstand. In Leipzig betrug der Leerstand mit knapp 60000 leerstehenden Wohneinheiten etwa $20 \%$ des Wohnungsbestands, in Dresden waren es mit
48000 Wohneinheiten rund $17 \%$ (Banse 2001, Effenberger 2001).

Die Leerstände waren in der zweiten Hälfte der 90er Jahre erheblich angestiegen: In Sachsen kam es in zweieinhalb Jahren zwischen 1995 und 1998 zu einer Verdopplung des Leerstands, in Leipzig stieg der Leerstand zwischen 1995 und 1999 um den Faktor 3,2. Zu den Ursachen hierfür zählen die Baufertigstellungen, Abwanderungen und die Veränderung von Wohnpräferenzen. Auch in Zukunft wird mit Leerständen zu rechnen sein. So rechnet die Kommission Wohnungswirtschaftlicher Wandel in den neuen Bundesländern in ihrem im Jahr 2000 veröffentlichten Bericht in 20 Jahren in Ostdeutschland mit einem Anstieg auf 2 Mio. leerstehende Wohneinheiten, „falls es zu keiner wirklich drastischen Veränderung der politischen Rahmenbedingungen und zu keiner Umsteuerung“ kommt (Banse 2001, Effenberger 2001, Petzold 2001).

Dennoch wird in einzelnen Wohnungsteilmärkten auch in den kommenden Jahren mit Zuwächsen zu rechnen sein. Wenngleich der bis zum Jahr 2015 prognostizierte Bedarf in Ostdeutschland im Vergleich zu westdeutschen Regionen insgesamt wesentlich geringer ausfällt (vgl. Wohnungsprognose 2015 des BBR in Zusammenarbeit mit dem IÖR), so wird es doch auch weiterhin in einigen Marktsegmenten zu Nachfragesteigerungen kommen. In Ostdeutschland konzentrieren sich diese vor allem im Bereich des Ein- und Zweifamilienhausbaus, während in Mehrfamilienhäusern ein deutlicher Bedarfsrückgang prognostiziert wird (Iwanow u.a. 2001).

Die bisherigen Entwicklungen haben erhebliche Konsequenzen: Immobilienpreise sind - teilweise im Zuge einer „Normalisierung" der zuvor überhitzten Märkte drastisch gesunken. Dies hat zu Entwertungsprozessen des Immobilienbestandes geführt und stellt insbesondere große Wohnungsunternehmen bzw. Wohnungsgenossenschaften vor große Probleme. Hinzu kommen Kosten des Leerstands (Effenberger 2001) und in vielen Fällen auch Altschulden.

Marktbereinigungen und Rückbau sind in dieser Situation nicht nur aus Gründen des städtebaulichen Umbaus angeraten, sondern erfüllen auch wohnungswirtschaftliche Funktionen der Preisstabilisierung (Stadtumbau Ost). Dabei sollte im Einzelfall allerdings geprüft werden, inwieweit Abriss erforderlich ist bzw. inwieweit die Umbauziele auch bereits durch eine "Versiegelung“ von Gebäuden (Reserve zur späteren Wiedernutzung, „Gebäudelager“) erreicht werden können (vgl. Deilmann 2001). 


\section{Steuerung durch Raumplanung?}

\section{Herausforderungen für die Planung}

Insgesamt ist im Hinblick auf die Bevölkerungsentwicklung seit mehr als einer Dekade ein deutliches West-Ost-Gefälle entstanden. Allerdings ist $\mathrm{zu}$ beachten, dass es sich bei der Schrumpfung um einen Megatrend mit teilräumlich dominanten Ausprägungen handelt, der sich nicht ausschließlich auf Ostdeutschland erstreckt, sondern immer stärker auch die westdeutschen Regionen erfasst. Da dies erst langsam in das Bewusstsein der Öffentlichkeit dringt, ist eine Steuerung der Schrumpfung wesentlich komplizierter und politisch schwieriger zu bewerkstelligen als eine Steuerung des Wachstums. Es ist zudem äußerst zweifelhaft, ob eine strukturelle Schrumpfung - wie vielfach angestrebt - durch die Umkehr negativer Trends in Wachstum bereits allein bewältigt werden kann.

Der Beirat für Raumordnung hat im Dezember 2001 in Folge der Vorlage des Raumordnungsberichts 2000 und der Raumordnungsprognose 2015 eine Veränderung der Planung angemahnt und dabei die folgenden Herausforderungen benannt (vgl. Beirat für Raumordnung 2001, S. 6 f.):

\section{- Raumentwicklungstendenzen neu bewerten:}

Dabei wird darauf hingewiesen, dass es der Beantwortung der Frage bedarf, „welche Orientierung bei dem 'Prinzip der Gleichwertigkeit der Lebensverhältnisse' zugrunde zu legen ist und wie dieses Prinzip längerfristig gehalten werden kann." In diesem Zusammenhang sei auch die Frage zu beantworten, zu welchem Preis die Gesellschaft bereit sei, ökologische Ausgleichsfunktionen in der Fläche, insbesondere in peripheren Räumen zu finanzieren. Zudem bedürfe das Prinzip der „Nachhaltigkeit“ noch der Operationalisierung und Umsetzung. Die Chancen, die es für eine Aufwertung der Raumordnung biete, seien noch nicht hinreichend genutzt worden. Hierzu bestehe dringender Handlungsbedarf.

\section{- Regionale Disparitäten zwischen Ost und West weiter abbauen:}

Wenn sich die bisherigen Trends der wirtschaftlichen Entwicklung fortsetzten, verfestigten sich heterogene Strukturen mit einem West-Ost-Gefälle. Während sich in Westdeutschland regionale Unterschiede auf relativ hohem Niveau einstellten, komme es in Ostdeutschland zu erheblichen „Verwerfungen zwischen strukturschwachen Regionen und Wohlstandsinseln in einigen wenigen Stadtregionen“. Die Raumordnung habe die Aufgabe, hier gegenzusteuern. Sie könne dies aber nur tun, wenn sie stärker als bisher regionale Entwick- lungspläne fordere und auf dieser Grundlage Sanierungs- und Entwicklungskonzepte initiiere und mit einem integrierten, abgestimmten Mitteleinsatz eine engere Verknüpfung von Raumplanung und Strukturpolitik erreiche.

\section{- Neue Auffangstrategien für den langfristigen Bevölkerungsrückgang entwickeln:}

Die Förderung der regionsspezifischen Möglichkeiten müsse im Vordergrund stehen. Hier sei insbesondere die Politik gefordert, einen Umdenkungsprozess einzuleiten und räumliche Auffangstrategien in Richtung auf ein Netz tragfähiger, mittlerer Zentren zu entwickeln. Der Beirat mahnt an, von realistischen Bevölkerungsprognosen auszugehen und den überzogenen lokalen Wettbewerb um Einwohner, zum Beispiel durch großzügige Baulandausweisungen, einzudämmen. Eine positive Bevölkerungsentwicklung dürfe nicht mehr der alles beherrschende Erfolgsfaktor für eine aktive Kommunalpolitik sein. Vielmehr müssten auch hier ausgewogene regionale Maßstäbe zur Sicherung von Mindeststandards angelegt werden.

\section{- Folgen des demographischen Rückgangs stärker berücksichtigen:}

Es bedürfe einer neuen Strategie von regionaler Strukturpolitik und Arbeitsförderung, die sich bewusst auf die Sicherung des Humankapitals als wichtigstem regionalen Entwicklungspotenzial ausrichte. Raumordnung und Regionalpolitik sollten verstärkt örtliche und regionale Schwerpunkte setzen. Eine Überprüfung der bisherigen Förderung „in der Fläche“ sei erforderlich. Im Hinblick auf den Wohnungsmarkt komme es darauf an, einerseits die Herausbildung differenzierter Wohnformen und eine lebenszyklusorientierte Durchmischung sozialer Strukturen in Wohnquartieren zu fördern und andererseits die Eigentumsbildung insbesondere in Ostdeutschland - zu unterstützen.

Die genannten Aspekte umreißen das Feld der zukünftigen Herausforderungen. Vor dem Hintergrund der hier dargestellten Entwicklungstendenzen lassen sich diese Punkte um folgende Aspekte erweitern:

\section{- Kleinteiligkeit von Entwicklungen stärker beachten:}

Schrumpfung und Wachstum liegen räumlich nahe beieinander. Gemeinden mit Wachstumstendenzen liegen unmittelbar neben schrumpfenden Gemeinden. Gemeinden mit einer ungünstigen Bevölkerungsstruktur befinden sich in Nachbarschaft zu Gemeinden mit relativ günstigen Strukturen. Sachgerechte Raumentwicklungsstrategien erfordern daher eine wesentlich kleinteiligere Raumbeobachtung. Entsprechende Instrumentarien sollten ausgebaut werden. 


\section{- Instrumente einer angepassten Raumfinanz- und Raumsozialpolitik entwickeln:}

Die aktuellen räumlichen Entwicklungstendenzen werden wesentlich von den im Raum wirkenden Instrumenten der Finanz- und Steuerpolitik beeinflusst. Diese sind nur unzureichend auf eine nachhaltige Raumentwicklung und den Ausgleich von Disparitäten ausgerichtet. Daher geht es in der Zukunft darum, die Wirkung von finanziellen Anreizen in ihrer räumlichen Dimension und im Hinblick auf raumsozialpolitische Ziele (funktionsorientierter Lasten-Nutzen-Ausgleich, Unterstützung peripherer Räume mit starken demographischen „Deformationen“) stärker zu beachten.

\section{- Einsparpotenziale durch geschicktes "Facility“-Management nutzen:}

Schrumpfung erfordert einerseits in zunehmendem Ausmaß eine Konzentration auf vorhandene Bestände, andererseits sind neue Bedarfe, die sich aus den Veränderungen der Bevölkerungsstruktur ableiten, zu befriedigen. Bei knappen Kassen ist dies nur durch ein geschicktes Management vorhandener Ressourcen möglich. Konzepte des „Facility“-Management sind im Hinblick auf ihre Tauglichkeit für die Raumentwicklungspolitik zu untersuchen.

\section{- Übersektorale Integration und gezielten Mitteleinsatz fördern:}

Schrumpfung hat in der Regel nicht nur Auswirkungen in einem Bereich, sondern ist Grundlage für veränderte Bedarfe, die sich gleichzeitig in mehreren Sektoren bemerkbar machen. Daher ist es nicht ausreichend, sektorale Antworten auf die Herausforderungen der Schrumpfung zu geben. Vielmehr sind integrierte Stadt- und Regionalentwicklungskonzepte erforderlich. Sie könnten - ähnlich wie zum Beispiel im Rahmen des Programms „Stadtumbau Ost“ - Grundlage für eine abgestimmte Förderpolitik und einen gezielteren Mitteleinsatz sein.

\section{- Ebenen, Verwaltungsgrenzen und Kompetenz- bereiche übergreifende Koordination und Kooperation stärken:}

Die Kleinteiligkeit von Entwicklungen macht deutlich, dass es sich bei den Folgen von Schrumpfung um Phänomene handelt, die sich weder von einer Planungsebene allein noch im Rahmen enger Verwaltungsgrenzen oder ressortbezogener Kompetenzbereiche zufriedenstellend bearbeiten lassen. Daher sollten übergreifende Koordinations- und Kooperationsprozesse intensiviert und ausgeweitet werden. Dem Staat kommt dabei eine rahmensetzende und initiierende Funktion zu. Er sollte Initiativfunktionen dort übernehmen, wo Kooperations- und Koordinationsstrukturen nur unzureichend entwickelt werden.

\section{Vom Wachstums- zum Schrumpfungsparadigma?}

Die Raumplanung ist in der Praxis noch nicht ausreichend auf Schrumpfungsprozesse vorbereitet. Dies wird durch einen Blick in jüngere Regionalpläne deutlich. Gleichwohl gibt es auch Ansätze in den Plänen, die entschiedene Reaktionen auf die neuen Herausforderungen erkennen lassen: So widmen sich Regionalpläne Problemen der Innenentwicklung, der Weiter-, Umund Nachnutzung, der Konversion und Nutzung von vorhandenen Standorten sowie eines modernen Flächenmanagements. Gleichwohl machen die Pläne noch zu wenig deutlich, wie diese Ziele regional operationalisiert werden können und welche Instrumente der Politik an die Hand gegeben werden sollen, um das Erreichen der Ziele zu überprüfen.

Die aktuellen Entwicklungstendenzen legen es vor diesem Hintergrund nahe, dass neben das traditionell wachstumsorientierte Planungsparadigma in Zukunft ein „Paradigma der Schrumpfung“ treten wird (vgl. Übersicht). Die wachstumsorientierte „Verteilung“ von Zuwächsen, die vorwiegend auf Neuausweisungen und Neubau orientierten Instrumente und die ordnungsorientierte Steuerung der Flächennutzung und baulichen Entwicklung allein reichen nicht aus, um wirksame Antworten für die Herausforderungen der Schrumpfung zu finden. Daher sind Planungsansätze stärker in Richtung auf ein „Schrumpfungsparadigma“ hin zu orientieren. 
Bernhard Müller: Regionalentwicklung unter Schrumpfungsbedingungen

Kennzeichen einer wachstums- und einer schrumpfungsorientierten Planung

\begin{tabular}{|l|l|}
\hline Wachstumsorientierte Planung & Schrumpfungsorientierte Planung \\
\hline $\begin{array}{l}\text { Wachstumsorientierung, „Verteilung“ von quantitativen } \\
\text { Zuwächsen (Einwohner, Arbeitsplätze usw.) }\end{array}$ & $\begin{array}{l}\text { Bestandsentwicklung, Stabilisierung, Revitalisierung, qualitative } \\
\text { Entwicklung (Wohnumfeld, Infrastruktur, Verkehr usw.) }\end{array}$ \\
\hline $\begin{array}{l}\text { Vorwiegend auf Neuerschließung von Flächen und Neubau } \\
\text { ausgerichtete baurechtliche und raumplanerische Instrumente; } \\
\text { Infrastrukturentwicklung als Vorleistung und Anreiz für } \\
\text { Investitionen }\end{array}$ & $\begin{array}{l}\text { Wiedernutzung von Flächen und Gebäuden, differenzierter } \\
\text { Rückbau, Anpassung von Infrastruktur an neue Bedürfnisse }\end{array}$ \\
\hline $\begin{array}{l}\text { Zuwachsorientierte Steuerung (Flächennutzung und bauliche } \\
\text { Entwicklung) }\end{array}$ & $\begin{array}{l}\text { Initiierung und Organisation von Rückbau, Sanierung und } \\
\text { Entwicklung bei knapperen finanziellen Ressourcen }\end{array}$ \\
\hline $\begin{array}{l}\text { Planung als Grundlage zur Verteilung von Zuwächsen, } \\
\text { Trennung von Raumfunktionen (Wohnen, Arbeiten usw.) }\end{array}$ & $\begin{array}{l}\text { Planung als Management von Prozessen der Schrumpfung, } \\
\text { kleinteilige funktionale Durchmischung }\end{array}$ \\
\hline $\begin{array}{l}\text { Ordnungsorientierte Steuerung der Flächennutzung und } \\
\text { baulichen Entwicklung, Ausweisungen von Siedlungsflächen, } \\
\text { Schutz von Freiflächen }\end{array}$ & $\begin{array}{l}\text { Strategische Planung und integrierte Konzepte, } \\
\text { Folgenabschätzungen, Umsetzung, Projekte, Nutzungsoptionen, } \\
\text { Aktivierung, vertragliche Regelungen, Effizienz }\end{array}$ \\
\hline $\begin{array}{l}\text { Interkommunaler Wettbewerb (Einwohner, Betriebe usw.), } \\
\text { sektorale Anreize, intersektorale Rahmensteuerung }\end{array}$ & $\begin{array}{l}\text { Interkommunale Kooperation, Ausgleichsregelungen, } \\
\text { Mehrebenenkooperation, intersektorale Koordination }\end{array}$ \\
\hline
\end{tabular}

Entwurf: Müller, B., 2002

\section{Steuerungsperspektiven der Raumplanung}

Die Raumplanung hat mit der Novelle des deutschen Bau- und Planungsrechts im Jahr 1998 erste Schritte in Richtung auf eine Umorientierung unternommen, die im Sinne der hier behandelten Herausforderungen und Zukunftsaufgaben nutzbar gemacht werden können. Es handelt sich dabei u.a. um

\section{- die engere Verzahnung von kommunaler und regionaler Planung.}

Folgen der Schrumpfung werden vor allem auf der lokalen Ebene wirksam. Kommunen haben in erhöhtem Maße finanzielle Lasten zu tragen und den infrastrukturellen Umbau zu bewältigen. Gleichwohl führt dies bei einer ausschließlich kommunalen und an den jeweiligen Gemeindegrenzen Halt machenden Perspektive zu suboptimalen Ergebnissen. Daher ist die regionale Ebene gefragt, über strategische Konzepte günstigere Rahmenbedingungen für die Bewältigung von Schrumpfungsprozessen und kleinräumigen Wachstums-Schrumpfungs-Disparitäten zu schaffen sowie konkrete interkommunale Planungen zu fördern. Die Instrumente der regionalen Flächennutzungsplanung und des regionalen Flächenmanagements sollten dabei in zunehmendem Maße nutzbar gemacht werden.

\section{- eine stärkere Umsetzungsorientierung der Regionalplanung.}

Die Regionalplanung muss sich stärker in Richtung auf eine Regionalentwicklung hin bewegen. Dies erfordert nicht nur klare und operationalisierbare Zielsetzungen, sondern kann auch darüber hinaus in dreifacher Hinsicht erfolgen:

- Erstens sollte die Regionalplanung grundsätzlich enger mit der Regionalentwicklung verknüpft werden. Dies ist u.a. in einigen Bundesländern bereits der Fall bzw. es erfolgen derzeit entsprechende Schritte in diese Richtung. Die Vorkehrungen des $\$ 13$ ROG, demzufolge regionale Entwicklungskonzepte eine wichtige Funktion bei der Verwirklichung der Raumordnungspläne haben, weisen in diese Richtung.

- Zweitens sollte die interkommunale Kooperation wesentlich gestärkt werden. Die in $\S 13$ ROG genannten Städtenetze sind ein positiver Schritt, reichen jedoch in dieser Hinsicht nicht aus, denn hier geht es um kleinräumige innerregionale Kooperationen in wesentlichen kommunalen Handlungsfeldern, in denen die Regionalplanung per se lediglich eine Rahmensteuerung betreiben kann, auf die sie aber über informelle Instrumente (Moderation, Mediation) wesentlich Einfluss nehmen kann. Die in Sachsen ausgewiesenen "nachbarschaftlichen“ zentralörtlichen Städteverbünde und landesplanerisch initiierte Kooperationsräume könnten hier einen nicht unwesentlichen Beitrag leisten. 
- Drittens kommt es auf engere Kooperationen zwischen öffentlichen und privaten Stellen (PublicPrivate-Partnership) an. Die Einführung der optionalen Öffentlichkeitsbeteiligung ( $\$ 7$ ROG) öffnet der Regionalplanung den Zugang zu diesem Feld. Gleichzeitig könnten raumordnerische Verträge eine wichtige Rolle bei der konkreten Umsetzung von raumordnungspolitisch bedeutsamen Projekten spielen (§ 13 ROG) (vgl. Spannowsky 1999).

\section{- eine Spezifizierung von Inhalten von Raumordnungsplänen:}

$\S 7$ ROG enthält Aussagen zu „Mindestinhalten“ von Raumordnungsplänen. Raumordnungspläne sollen Festlegungen zur Raumstruktur, d.h. zur Siedlungsstruktur, zur Freiraumstruktur und zu den zu sichernden Standorten und Trassen für Infrastruktur, sowie zu raumbedeutsamen Planungen und Maßnahmen enthalten und können Vorrang-, Vorbehalts- und Eignungsgebiete festlegen. Wenngleich die damit verbundene Standardisierung von Inhalten einerseits grundsätzlich als ein Fortschritt in der Raumplanung angesehen werden kann, so ist andererseits doch auch festzustellen, dass dabei kaum Elemente aufgeführt werden, die sich explizit für eine Steuerung von Schrumpfungsprozessen anbieten. Teilweise wären bereits Ergänzungen bestehender Festlegungen ausreichend. Zwei Beispiele sollen dies belegen:

\section{(1)}

Defizite hinsichtlich der Steuerungswirksamkeit des Zentrale-Orte-Konzepts sind bereits oben angesprochen worden. Hier könnte es sich zum Beispiel im Hinblick auf Schrumpfungsprozesse als vorteilhaft erweisen, in stärkerem Maße zentralörtliche Kooperationsräume auszuweisen und entsprechend $z u$ instrumentieren (vgl. Forschungsverbund Stadt und Region in Sachsen 2002).

\section{(2)}

Das ROG schafft zwar Voraussetzungen zur Festlegung von Sanierungs- und Entwicklungsgebieten, diese werden jedoch als eine Kategorie der Freiraumstruktur angesehen. Modellvorhaben der Raumordnung haben gezeigt, dass eine solche Beschränkung nicht zweckmäßig ist und dass Sanierungs- und Entwicklungsgebiete eher im Sinne der zum Beispiel in Sachsen (und in ähnlicher Art auch in Thüringen) ausgewiesenen Gebiete mit besonderen Entwicklungs-, Sanierungs- und Förderungsaufgaben interpretiert werden sollten. Allerdings sollten diese - im Unterschied zur bisherigen Praxis - eher für Räume einer mittleren Maßstabsebene spezifiziert werden (vgl. Müller, Rathmann, Wirth 2002).

\section{Neuinterpretation des Gegenstromprinzips}

Die genannten Ansätze bieten eine Grundlage, Schrumpfungsprozesse raumordnerisch besser zu bearbeiten. Allerdings reichen sie nicht aus, um eine umfassende Verbesserung des raumordnerischen Instrumentariums zum Umgang mit Schrumpfung zu erreichen. In der Regel sind nicht nur die Kommunen, sondern auch die Regionen allein mit der Bewältigung der Folgeprobleme von Schrumpfung überfordert. Daher wird es in der Zukunft auf eine Neuinterpretation des "Gegenstromprinzips“ ankommen. Damit ist - in gewisser Analogie zum Gegenstromprinzip in der Raumplanung und unter Verwendung des Ansatzes einer "anreizorientierten Mehrebenensteuerung“ (Müller 2000) bzw. mit Bezug auf Ansätze einer „parametrischen Steuerung" (Fürst 2001) - das Zusammenspiel einer Rahmensteuerung "von oben“ und einer Koordination „von unten“ gemeint. Es ist durch die folgenden Elemente geprägt:

\section{- Festlegung von Qualitätskriterien für Pläne und Programme (Steuerung von oben):}

Der staatlichen Rahmensteuerung kommt die Aufgabe zu, im Dialog mit den Betroffenen (Regionen und Kommunen) Qualitätskriterien für Programme und Pläne festzulegen. Dies muss im Hinblick auf die Steuerung von Schrumpfung deutlich über die Festlegung von Mindestinhalten (im Sinne des ROG) hinausgehen. Insbesondere ist dabei auf die spezifische Situation von Schrumpfungsregionen einzugehen. Qualitätskriterien stellen einen Rahmen dafür dar, welche Aufgaben von den Regionen zu bearbeiten sind und welche Elemente in Lösungskonzepten Beachtung finden sollen. Rahmensteuerung "von oben“ umfasst jedoch nicht die direkte inhaltliche Steuerung oder Beeinflussung der Inhalte solcher Konzepte.

\section{- Wettbewerbsorientierte Vergabe von Fördermitteln mit Prozessunterstützung.}

Schrumpfungsprobleme können weder von Kommunen noch von Regionen allein gelöst werden. In der Regel bedürfen sie staatlicher Unterstützung zur Förderung von Maßnahmen zur Beseitigung von Entwicklungshemmnissen und zur Abfederung von negativen Entwicklungen. Diese sollte jedoch weniger nach dem „Gießkannenprinizip“ als nach einem Wettbewerbsprinzip vergeben werden. Dabei sollte der Qualität vorgelegter Konzepte ein zentraler Stellenwert zukommen. Um gleiche Wettbewerbschancen herzustellen und eventuelle Wettbewerbsnachteile von Regionen auszugleichen, ist eine Prozessunterstützung bei der Entwicklung von Konzepten (Moderation, Beratung) erforderlich. 


\section{- Integrierte Entwicklungskonzepte (Steuerung von unten):}

Integrierte (Stadt- oder Regional-) Entwicklungskonzepte können der Steuerung „,on unten“ dienen, indem sie klare Prioritäten für die räumliche Entwicklung formulieren, die sich am schrumpfungsbedingten Handlungsbedarf orientieren, die für Problemlösungen bedeutsamen Akteure ,an einen Tisch“ bringen und diese zu sektorübergreifendem Handeln animieren. Ein Qualitätskriterium für die Beurteilung von Konzepten könnte sein, inwieweit es den Kommunen und Regionen gelingt, dies bereits bei der Ausarbeitung von Ideenkonzepten zu erreichen.

\section{- Operationalisierung von Zielen:}

Regionalentwicklung ist auf umsetzungsorientierte oder für eine Umsetzung interpretierbare Ziele der Planung angewiesen. Häufig erfüllen Raumordnungspläne dies nur unzureichend sowohl im Hinblick auf den Grad der Konkretisierung von Zielen als auch auf den Kreis der Adressaten, an die sich die Ziele richten. Raumordnungspläne erlauben daher kaum ein zielorientiertes Monitoring von Raumentwicklungsprozessen. Schrumpfung bedarf jedoch kleinteiliger und permanenter „Beobachtung“, um zeitnah auf die jeweiligen Anforderungen reagieren zu können.

\section{- Szenarien und Folgenabschätzungen:}

Maßnahmen, die auf eine Bewältigung von Schrumpfungsproblemen abzielen, sind in ihren kurz-, mittelund langfristigen Wirkungen nicht immer eindeutig. So können zum Beispiel Abrissprogramme zu Bereinigungen auf dem Wohnungsmarkt führen, aber auch bauliche Reserven, die mittelfristig anderen Nutzungen zugeführt werden könnten, vernichten. Hier stellt sich zum Beispiel die Frage, inwieweit die zeitlich befristete "Versiegelung“ von Gebäuden zum Offenhalten von Optionen beitragen kann. Zudem können Abrissprogramme zu ungewollten Problemen von ,sozialer und räumlicher Entwurzelung" führen. Dies unterstreicht die Notwendigkeit, bereits im Vorfeld auf dem Weg von Szenarien mögliche Zukünfte der Entwicklung zu skizzieren und einzuschätzen. Auch dies könnte als ein wesentliches Kriterium zur Beurteilung von lokalen und regionalen Entwicklungskonzepten angesehen werden.

\section{- Kleinteiliges Monitoring.}

Schrumpfung manifestiert sich kleinräumig und „schleichend“. Daher ist eine kontinuierliche Beobachtung von Entwicklungsprozessen unabdingbar, wenn man zielgerichtet und sachgerecht auf Schrump- fungsprobleme reagieren will. Das Monitoring sollte insbesondere nicht nur Phänomene von Schrumpfung erfassen, sondern auch Ursachenanalysen erlauben.

\section{- Information und öffentliche Debatte:}

Schrumpfungsprozesse und ihre Folgen bedürfen einer offensiven Informationsstrategie. Schrumpfungsbedingte Probleme der lokalen und regionalen Entwicklung können nämlich in der Regel nicht von öffentlichen Stellen allein gelöst werden. Vielmehr erfordern sie von allen Gruppen der Gesellschaft Kenntnisse über ihre Ursachen und Wirkungen und ein Engagement bei ihrer Bewältigung. Eine sachlich geführte öffentliche Debatte ist notwendig, um Verständnis für - gegebenenfalls auch einschneidende - Maßnahmen zu erzeugen. Es ist Aufgabe der Politik, eine solche Debatte zu initiieren.

Dieser Ansatz ist im Grunde nicht neu. Er wird vielmehr in seinen Grundzügen seit den 90er Jahren - insbesondere im Rahmen von Wettbewerben - implizit zumindest ansatzweise angewendet. Beispiele hierfür sind (vgl. Müller 1999, Adam, Wiechmann 1999, Baitsch, Müller 2001):

- die deutliche Betonung kooperativer Ansätze in Landes- und Regionalplänen, die auf einen möglichst sparsamen Ressourceneinsatz und die Bewältigung von Problemen im interkommunalen Verbund gerichtet sind,

- Initiativen zur engeren Kopplung von Regionalplanung und Regionalentwicklung, so etwa im Zuge der Anwendung von Förderrichtlinien zur Regionalentwicklung (z.B. Regionalisierung der Strukturpolitik in Nordrhein-Westfalen, FR-Regio in Sachsen) oder struktureller bzw. organisatorischer Veränderungen der Regionalplanung (z.B. in Bayern, NordrheinWestfalen, Thüringen, Sachsen-Anhalt und Mecklenburg-Vorpommern),

- die Förderung der Kooperation in Städtenetzen und Städteverbünden sowie die Festlegung von (zentralörtlichen) Kooperations- bzw. Funktionsräumen, die auf eine bessere Abstimmung zwischen den Kommunen und interkommunale Lernprozesse (teilweise mit Ansätzen von Benchmarking) abzielen,

- die Erarbeitung von regionalen Entwicklungs- und Handlungskonzepten, die insbesondere an der Bewältigung lokaler und regionaler Entwicklungsprobleme - in Ostdeutschland und im Ruhrgebiet nicht selten auch vor dem Hintergrund von Schrumpfungsfolgen - ansetzen,

- die Wettbewerbe „Städte der Zukunft“ (BMVBW I BBR), „Stadt 2030" (BMBF) und „Stadtumbau Ost“ 
(BMVBW), die auf die Kommunen abzielen und teilweise explizit (Stadtumbau Ost) an der Bewältigung von Schrumpfungsproblemen ansetzen,

- die Wettbewerbe "Regionen der Zukunft“ (BMVBWI BBR) und "Regionen aktiv" (BMVL), die zumindest indirekt in den meisten ostdeutschen Regionen als Instrument zur Auseinandersetzung mit Problemen der Transformation genutzt worden sind, wenngleich in der Regel nicht auf der Grundlage eines "Schrumpfungsparadigmas",

- das Modellvorhaben zu „Sanierungs- und Entwicklungsgebieten“, in dessen Rahmen Umbauprozesse in drei Modellregionen - in einem Fall, dem Aktionsraum um Johanngeorgenstadt, mit einem deutlich schrumpfungsbetonten Ansatz (Rückbau) - initiiert wurden,

- der Wettbewerb „Innoregio“ (BMBF) zur Förderung einer innovationsorientierten Regionalentwicklung, der sich auf Ostdeutschland beschränkte und darauf abzielte, Innovationsdefizite und -hindernisse zu beseitigen und dabei insbesondere Privaten eine tragende Rolle beimaß,

- die IBA Emscher Park und IBA Fürst-Pückler-Land, in deren Rahmen es um den Umbau von Regionen und neue lokale und regionale Entwicklungsimpulse geht.

In vielen der genannten Fälle war die Regionalplanung im Hinblick auf ein aktives Engagement noch relativ zurückhaltend und zögerlich, obwohl die Rahmenbedingungen dies nicht unbedingt nahe legten (Adam, Wiechmann 1999). Ein offensiveres Verhalten wäre in der Zukunft anzustreben.

Raumplanung und Regionalentwicklung könnten dann dazu beitragen, dass die „Transformationslücke“ in Deutschland verringert wird. Dabei gilt es, sich offensiver auf den Umgang mit Schrumpfung einzustellen. Dazu bedarf es aber eines offenen Umgangs mit Schrumpfungsproblemen, kreativer Problemlösungsansätze und vor allem eines schnellen Handelns.

\section{Anmerkung}

* Dem Beitrag liegen Manuskripte zu Vorträgen über Schrumpfungsprozesse zu Grunde, die der Autor beim Institut für Städtebau der Deutschen Akademie für Städtebau und Landesplanung und beim Hauptausschuss des Deutschen Städtetages in Berlin gehalten hat. Der Autor dankt seinen Mitarbeitern, den Herren Gerard Hutter, Stefan Siedentop und Thorsten Wiechmann, für wertvolle Anregungen.

\section{Literatur}

Adam, B.; Wiechmann, Th. (1999): Die Rolle der Raumplanung in regionalen Agenda-Prozessen. In: Informationen zur Raumentwicklung,. Heft $9 / 10$

Akademie für Raumforschung und Landesplanung (ARL) (1998): Methoden und Instrumente der räumlichen Planung. Hannover

Baitsch, C.; Müller, B. (Hrsg.) (2001): Moderation in regionalen Netzwerken. München

Banse, J. (2001): Bevölkerungsentwicklung und Wohnungsangebot. In: Petzold, $\mathrm{H}_{\text {; }}$ Reichart, Th. (Hrsg.): Wohnungsmarkt in Sachsen im Spannungsfeld von Schrumpfung und Wachstum. Dresden

Beirat für Raumordnung (2001): Konsequenzen und kritische Würdigung des Raumordnungsberichts 2000

Bucher, Hansjörg (2000): Entwicklungstrends der ostdeutschen Bevölkerungs- und Siedlungsstruktur. In: Bundesamt für Bauwesen und Raumordnung (BBR): Regionale Aspekte des wirtschaftlichen und sozialen Wandels in den neuen Ländern. Regionalbarometer neue Länder. Vierter zusammenfassender Bericht. Bonn

Danielzyk, R.; Müller, B.; Priebs, A.; Wirth, P. (Hrsg.) (2000): Sanierung und Entwicklung in Ostdeutschland - regionale Strategien auf dem Prüfstand. Dresden

Deilmann, C. (2001); Überkapazitäten im Wohnungsbau als ökologische Herausforderung. In: Petzold, H ; Reichart, T. (Hrsg.) : Wohnungsmarkt in Sachsen im Spannungsfeld von Schrumpfung und Wachstum. Dresden

Doehler, M.; Reuter, I (2001): Schrumpfung planen? In: wohnbund-informationen. Heft 3, Info-Spezial: „Ost - schrumpfende Stadt"

Effenberger, K.-H. (2001): Wohnungsleerstand und Wohnungsversorgung. In: Petzold, H ; Reichart, T. (Hrsg.) : Wohnungsmarkt in Sachsen im Spannungsfeld von Schrumpfung und Wachstum. Dresden

Europäische Kommission (2001): Einheit Europas. Solidarität der Völker. Vielfalt der Regionen. Zweiter Bericht über den wirtschaftlichen und sozialen Zusammenhalt. Luxemburg

Forschungsverbund Stadt und Region in Sachsen (2002): Oberzentrale Kooperationsräume in Sachsen. Dresden (unveröffentl. Forschungsbericht)

Fürst, D. (2001): Parametrische Steuerung. Hannover (unveröffentl. Manuskript)

Hannemann, Ch. (2000): Zukunftschance Schrumpfung - Stadtentwicklung in Ostdeutschland - eine Skizze. In: Hager, F.; Schenkel, W. (Hrsg.) (2000): Schrumpfungen. Chancen für ein anderes Wachstum. Ein Diskurs der Natur- und Sozialwissenschaften. Berlin U. a., S. $99-105$ 
Irmen, E., Maretzke, S., Milbert, A. (2001): Regionale Entwicklungspotenziale in den neuen Ländern - Erwartung und Wirklichkeit. In: Informationen zur Raumentwicklung, Heft $2 / 3$

Iwanow, I., Metzmacher, M.; Schmidt, R.; Waltersbacher, M. (2001): Zukünftige Wohnungsnachfrage und Neubaubedarf in Ost- und Westdeutschland. Dresden. = IÖR-Texte 133

Mackensen, R. (1984): Leben im Jahre 2000 und danach. Berlin

Müller, B. (1999): Kooperative Entwicklungsansätze in Ostdeutschland: Von der Raumordnung zur Regionalentwicklung. In: Informationen zur Raumentwicklung, Heft 9/10

Müller, B. (2000): Regionen der Zukunft - Wege zur Wettbewerbsfähigkeit. In: Wolf, K.; Tharun, E. (Hrsg.): „Rhein-Main“ im Europa der Regionen. Frankfurt a.M.

Müller, B.; Rathmann, J.; Wirth, P. (2002): Sanierungs- und Entwicklungsgebiete. Berlin

Petzold, H. (2001): Strategien der Stadtentwicklung für schrumpfende Städte - Überlegungen zum Umgang mit dem Wohnungsleerstand. In: Petzold, H ; Reichart, T. (Hrsg.): Wohnungsmarkt in Sachsen im Spannungsfeld von Schrumpfung und Wachstum. Dresden

Siedentop, St. (1998): Verkehrsvermeidende Siedlungsentwicklung unter Schrumpfungsbedingungen. Ausgangsüberlegungen zur Entwicklung siedlungs-struktureller Konzepte für die ostdeutschen Stadtregionen. In: Wolf, K.; Tharun, E. (Hrsg.): Verkehrsplanung und städtebauliche Entwicklung. Frankfurt a.M.
Siedentop, S. et al. (2002): Siedlungsstrukturelle Veränderungen im Umland der Agglomerationsräume (Entwurf des Abschlussberichts). Dresden (unveröffentl. Manuskript)

Spannowsky, W. (1999): Verwirklichung von Raumordnungsplänen durch vertragliche Vereinbarungen Bonn. $=$ BBR, Forschungen, Heft 93

Wiechmann, Th. (1998): Vom Plan zum Diskurs? Anforderungsprofil, Aufgabenspektrum und Organisation regionaler Planung in Deutschland. Baden-Baden

Winkel, R. (2000): Kooperative Umlandverbünde - Eine Möglichkeit zur Fortentwicklung des Konzeptes der Zentralen Orte. In: Der Landkreis, 2000, H. 10

Winkel, R. (2001): Die Bevölkerungsentwicklung verändert die Anforderungen an die Siedlungsentwicklung. In: Raumforschung und Raumordnung, Heft 1

Wood, G. (1994): Die Umstrukturierung Nordost-Englands wirtschaftlicher Wandel, Alltag und Politik in einer Altindustrieregion. Dortmund

Prof. Dr. Bernhard Müller

Institut für ökologische

Raumentwicklung e.V. (IÖR)

Weberplatz 1

01217 Dresden

E-Mail: B.Mueller@ioer.de 